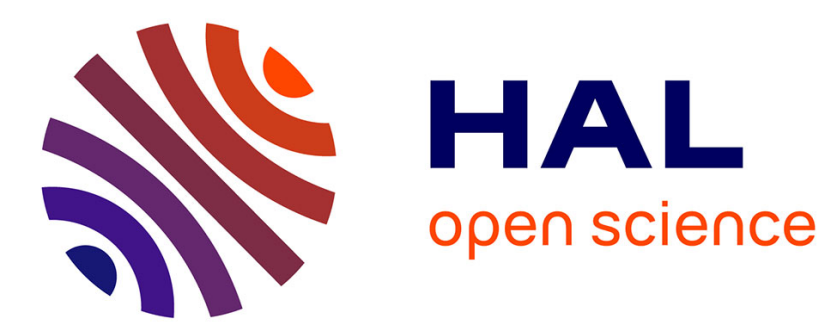

\title{
A Note on Residual Stress, Lattice Orientation and Dislocation Density in Crystalline Solids
}

\author{
David Steigmann, Raymond W. Ogden
}

\section{To cite this version:}

David Steigmann, Raymond W. Ogden. A Note on Residual Stress, Lattice Orientation and Dislocation Density in Crystalline Solids. Journal of Elasticity, 2012, 109 (2), pp.275-283. hal-00787094

\section{HAL Id: hal-00787094 \\ https://hal.science/hal-00787094}

Submitted on 11 Feb 2013

HAL is a multi-disciplinary open access archive for the deposit and dissemination of scientific research documents, whether they are published or not. The documents may come from teaching and research institutions in France or abroad, or from public or private research centers.
L'archive ouverte pluridisciplinaire HAL, est destinée au dépôt et à la diffusion de documents scientifiques de niveau recherche, publiés ou non, émanant des établissements d'enseignement et de recherche français ou étrangers, des laboratoires publics ou privés. 


\title{
A Note on Residual Stress, Lattice Orientation and Dislocation Density in Crystalline Solids
}

\author{
David J. Steigmann · Ray W. Ogden
}

\begin{abstract}
The theory of residually stressed crystals is discussed in the context of the finite deformation theory of elastic-plastic solids. Here residual stress is assumed to be due to the prior plastic flow of a single crystal. On the basis of recent experiments, as well as the structure of the underlying theory, we advance the view that the lattice orientation field should be regarded as data. This stands in contrast to the classical theory in which dislocation density is assigned.
\end{abstract}

Keywords Residual stress · Lattice orientation · Dislocation density · Crystalline solids

\section{Introduction}

In this note we revisit the static residual stress problem for metallic crystals that have undergone prior plastic flow. The geometrically linear version of this problem received definitive treatment in the work of Kröner and others [1-3], in which methods to determine the stress field generated by an assigned field of dislocation density were developed. Here the problem is posed in terms of the modern finite deformation theory of elastic-plastic response, as presented in $[4,5]$, specialized to the case of small elastic strain with finite lattice rotation. The basic theory is connected to contemporary experiments designed to quantify the dislocation density.

We use standard notation such as $\mathbf{A}^{\mathrm{T}}, \mathbf{A}^{-1}, \mathbf{A}^{*}, \operatorname{dev} \mathbf{A}, \operatorname{tr} \mathbf{A}$ and $J_{\mathbf{A}} \equiv \operatorname{det} \mathbf{A}$. These are, respectively, the transpose, the inverse, the adjugate, the deviatoric part, the trace and the determinant of a second-order tensor $\mathbf{A}$. The notation Sym is used to denote the linear subspace

D.J. Steigmann

Department of Mechanical Engineering, University of California, Berkeley, CA 94720, USA

R.W. Ogden (两)

School of Engineering, University of Aberdeen, Aberdeen AB24 3UE, UK

e-mail: r.ogden@abdn.ac.uk 
of symmetric tensors. The tensor product of two vectors is indicated by interposing the symbol $\otimes$, and the Euclidean inner product of tensors $\mathbf{A}$ and $\mathbf{B}$ is denoted by $\mathbf{A} \cdot \mathbf{B}=\operatorname{tr}\left(\mathbf{A B}^{\mathrm{T}}\right)$; the associated norm is $|\mathbf{A}|=\sqrt{\mathbf{A} \cdot \mathbf{A}}$. In terms of orthonormal components, $\mathbf{A} \cdot \mathbf{B}=A_{i j} B_{i j}$, wherein the usual summation rule for repeated indices is implied. For a fourth-order tensor $\mathcal{A}$, the notation $\mathcal{A}[\mathbf{B}]$ stands for the second-order tensor with orthonormal components $\mathcal{A}_{i j k l} B_{k l}$. Finally, D is used to denote the gradient of a vector field with respect to its argument.

\section{Basic Theory}

The variables that characterize an elastic-plastic solid in the static purely mechanical theory are the deformation $\chi(\mathbf{x})$ and the plastic deformation tensor $\mathbf{K}(\mathbf{x})$, where $\mathbf{x}$ is the position of a material point in a fixed reference placement $\kappa_{\mathrm{r}}$ of the body. The values $\mathbf{y}=\chi(\mathbf{x})$ are the positions of these points after deformation and generate the deformed placement $\kappa_{\mathrm{d}}$ of the body as $\mathbf{x}$ ranges over $\kappa_{\mathrm{r}}$. As is usual, we suppose positions in $\kappa_{\mathrm{r}}$ and $\kappa_{\mathrm{d}}$ to be in one-toone correspondence and thus the deformation gradient, $\mathbf{F}=\mathrm{D} \chi$ to be invertible. The elastic deformation is then defined through the tensor

$$
\mathbf{H}=\mathbf{F K} \text {. }
$$

Note that the measure of plastic deformation used most frequently in the literature corresponds to our $\mathbf{K}^{-1}$. We take $\kappa_{\mathrm{r}}$ to be a kinematically possible configuration of the body, so that $J_{\mathbf{F}} \equiv \operatorname{det} \mathbf{F}>0$. The elastic deformation gradient is assumed to be the value of the deformation gradient in the absence of plastic deformation, this condition being identified by $\mathbf{K}=\mathbf{I}$, the identity for second-order tensors. We therefore impose $J_{\mathbf{H}}>0$ and deduce that $J_{\mathbf{K}}>0$. We shall also make use of an intermediate configuration, denoted $\kappa_{\mathrm{i}}$, in which $\mathbf{H}=\mathbf{I}$.

The elastic strain energy per unit volume of $\kappa_{\mathrm{d}}$ is denoted $\psi(\mathbf{H})$, and we assume that the material of the body is uniform in the sense that this strain-energy function does not depend explicitly on $\mathbf{x}$. The strain-energy function referred to $\kappa_{\mathrm{i}}$ is

$$
W(\mathbf{H})=J_{\mathbf{H}} \psi(\mathbf{H}) .
$$

We also define the reduced strain energy $\hat{W}$ by

$$
\hat{W}(\mathbf{C})=W(\mathbf{H}),
$$

where

$$
\mathbf{C}=\mathbf{H}^{\mathrm{T}} \mathbf{H}
$$

is the right elastic Cauchy-Green deformation tensor. That the strain energy depends on $\mathbf{H}$ via $\mathbf{C}$ follows from the symmetry of the Cauchy stress $\mathbf{T}$, related to $\mathbf{P}$ by $\mathbf{P}=\mathbf{T F}^{*}$ (see, for example, [6]). This, of course, is equivalent to $(5)_{2}$.

The local equations of equilibrium in the case of negligible body forces are

$$
\operatorname{Div} \mathbf{P}=\mathbf{0}, \quad \mathbf{P F}^{\mathrm{T}} \in \mathrm{Sym} \quad \text { in } \kappa_{\mathrm{r}},
$$

where Div is the referential divergence (the divergence with respect to $\mathbf{x}$ ),

$$
\mathbf{P}=\mathbf{F} \boldsymbol{\Pi}
$$


is the first Piola-Kirchhoff stress, and $\Pi$ is the second Piola-Kirchhoff stress relative to $\kappa_{\mathrm{r}}$. This is related to the corresponding stress $\mathbf{S}=\hat{\mathbf{S}}(\mathbf{C})$ relative to $\kappa_{\mathrm{i}}$ by

$$
\Pi=J_{\mathbf{K}}^{-1} \mathbf{K S K}^{\mathrm{T}},
$$

where

$$
\hat{\mathbf{S}}(\mathbf{C})=2 \frac{\partial \hat{W}}{\partial \mathbf{C}} .
$$

The strain-energy function is subject to the restriction

$$
\hat{W}(\mathbf{C})=\hat{W}\left(\mathbf{Q}^{\mathrm{T}} \mathbf{C Q}\right)
$$

associated with the transformation $\mathbf{H} \rightarrow \mathbf{H Q}$, where the rotation tensor $\mathbf{Q}$ is an arbitrary element of the symmetry group for the material, i.e. the symmetry group relative to the elastically undistorted configuration $\kappa_{\mathrm{i}}$ (see [4] for further discussion). In particular, material uniformity implies that this restriction is effective, with the same symmetry group, at every material point. Using (8), it is straightforward to show that $\mathbf{S} \rightarrow \mathbf{Q}^{\mathrm{T}} \mathbf{S Q}$ under symmetry transformations and hence that $\mathbf{T}$ is invariant.

The system (5) is augmented by suitable boundary data; in the standard case these are

$$
\chi(\mathbf{x}) \text { and } \mathbf{t}(\mathbf{x}) \text { assigned on } \partial \kappa_{\mathrm{r}(\boldsymbol{x})} \text { and } \partial \kappa_{\mathrm{r}(\mathbf{t})} \text {, respectively, }
$$

where $\partial \kappa_{\mathrm{r}(\boldsymbol{x})}$ and $\partial \kappa_{\mathrm{r}(\mathbf{t})}$ are complementary parts of the boundary $\partial \kappa_{\mathrm{r}}$ of $\kappa_{\mathrm{r}}$, and

$$
\mathbf{t}=\mathbf{P n}
$$

is the traction, $\mathbf{n}$ being the exterior unit normal to $\partial \kappa_{\mathrm{r}}$.

The elastic (lattice) strain

$$
\mathbf{E}=\frac{1}{2}(\mathbf{C}-\mathbf{I})
$$

and the true dislocation density

$$
\boldsymbol{\alpha}=J_{\mathbf{K}} \mathbf{K}^{-1} \operatorname{Curl}\left(\mathbf{K}^{-1}\right)
$$

are the relevant state variables [5, 7]. Here, Curl is the referential curl operation defined in terms of the usual vector operation by

$$
(\operatorname{Curl} \mathbf{A}) \mathbf{c}=\operatorname{Curl}\left(\mathbf{A}^{\mathrm{T}} \mathbf{c}\right)
$$

for any fixed vector c. We note in passing that an alternative definition of the operation of Curl on a second-order tensor is sometimes adopted in the literature, in which $\mathbf{A}^{\mathrm{T}}$ replaced by $\mathbf{A}$ on the right-hand side of the above.

The dislocation measure $\alpha$ is true in the sense that it is invariant under smooth variations of the spatial and reference configurations $\kappa_{\mathrm{d}}$ and $\kappa_{\mathrm{r}}$, respectively, and is thus intrinsic to the state of the material [7]. For this reason the (referential) gradient of the plastic deformation necessarily manifests itself in constitutive equations via the true dislocation density.

In view of our interest in metallic crystals, it is appropriate to restrict the elastic strain to be small. Accordingly, the strain energy and stress are approximated by

$$
\hat{W}=\tilde{W}(\mathbf{E})=\frac{1}{2} \mathbf{E} \cdot \mathcal{C}[\mathbf{E}], \quad \mathbf{S}=\tilde{\mathbf{S}}(\mathbf{E})=\mathcal{C}[\mathbf{E}]
$$


respectively, where $\mathcal{C}$ is the fixed fourth-order tensor of elastic moduli pertaining to the undistorted crystal, possessing the usual major and minor symmetries. We assume the strainenergy to be a positive-definite function and thus $\mathcal{C}$ to be positive definite on Sym. The assumption of small elastic strain is consistent with the presumed cessation of plastic flow, yielding a static distribution $\mathbf{K}(\mathbf{x})$. This implies that the elastic strain (or the stress) belongs to the interior of the elastic range of the material; the restriction is then justified in the case of metallic crystals.

The foregoing model applies to single crystals. In crystal elasticity theory, fixed linearly independent lattice vectors $\mathbf{I}_{i}, i \in\{1,2,3\}$, associated with an undistorted stress-free state of the crystal, are mapped to their images $\mathbf{d}_{i}$ in $\kappa_{\mathrm{d}}$ in accordance with the Cauchy-Born hypothesis. That is, they are regarded as material vectors. Here, as in [1], we invoke this hypothesis for the elastic deformation. Thus, we assume that $\mathbf{d}_{i}=\mathbf{H I}_{i}$, where $\mathbf{I}_{i}$ are the lattice vectors in $\kappa_{\mathrm{i}}$. The $\mathbf{d}_{i}$ are observable in principle. In practice it is their duals $\mathbf{d}^{i}$ that are measured in X-ray diffraction experiments [8].

It follows from (1) that $\mathbf{d}_{i}=\mathbf{F} \mathbf{r}_{i}$, where $\mathbf{r}_{i}=\mathbf{K} \mathbf{l}_{i}$ are the lattice vectors in $\kappa_{\mathrm{r}}$. The plastic deformation is then given by $\mathbf{K}=\mathbf{r}_{i} \otimes \mathbf{l}^{i}$, where the $\mathbf{l}^{i}$ are the duals of the $\mathbf{l}_{i}$. To see this we write $\mathbf{K}=\mathbf{K I}$ with $\mathbf{I}=\mathbf{l}_{i} \otimes \mathbf{l}^{i}$. It follows similarly that $\mathbf{H}=\mathbf{d}_{i} \otimes \mathbf{l}^{i}$. We assume the $\mathbf{I}_{i}$ associated with a material point to be fixed material vectors. These are the lattice vectors of the ideal undistorted stress-free lattice. In practice they are specified as data. Further, they are uniform in the case of a materially uniform body. In effect, the set of lattice vectors is treated as an assigned property of both the material and the body.

It is crucial that the undistorted, stress-free lattice $\left\{\mathbf{I}_{i}\right\}$, associated with the local configuration $\kappa_{\mathrm{i}}$, be carefully distinguished from a global reference configuration $\kappa_{\mathrm{r}}$. The latter need only be a fixed placement that could, in principle, be occupied by the body, and need not be stress free, whereas the former is an intrinsic property, modulo orientation, of the ideal crystal. In materially uniform bodies such as single crystals, the same undistorted lattice is attached to every material point of the crystal. The map of $\left\{\mathbf{I}_{i}\right\}$ via $\mathbf{K}$ then yields a distorted, possibly non-uniform lattice $\left\{\mathbf{r}_{i}\right\}$, which is simply the image in $\kappa_{\mathrm{r}}$ of the distorted-and hence stressed-observable lattice $\left\{\mathbf{d}_{i}\right\}$ in $\kappa_{\mathrm{d}}$. The stress $\Pi$ in $\kappa_{\mathrm{r}}$ is then non-zero in general, being simply the pull-back by $\mathbf{F}^{-1}$ to the reference configuration of the stress in $\kappa_{\mathrm{d}}$ induced by the elastic deformation that maps $\left\{\mathbf{I}_{i}\right\}$ to $\left\{\mathbf{d}_{i}\right\}$. In the same way the stress $\mathbf{S}$ is the pull-back by $\mathbf{H}^{-1}$ of this stress to $\kappa_{\mathrm{i}}$.

The foregoing discussion about lattices is based on Noll's concept of materially uniform bodies. Our interpretation of this concept follows that described in [9] where Noll's theory is used as the conceptual foundation for the analysis of a variety of inelastic phenomena, including plasticity.

\section{The Residual Stress Problem}

To characterize the state of the material in the (distorted) reference placement $\kappa_{\mathrm{r}}$, we evaluate (6) at $\mathbf{F}=\mathbf{I}$, obtaining $\mathbf{H}=\mathbf{K}$ and

$$
\mathbf{P}=\Pi(\mathbf{x})
$$

with

$$
\Pi=J_{\mathbf{K}}^{-1} \mathbf{K} \tilde{\mathbf{S}} \mathbf{K}^{\mathrm{T}},
$$

where

$$
\tilde{\mathbf{S}}(\mathbf{x})=\frac{1}{2} \mathcal{C}\left[\mathbf{K}^{\mathrm{T}} \mathbf{K}-\mathbf{I}\right]
$$


is the lattice stress $\mathbf{S}$. Here the dependence on $\mathbf{x}$ is due entirely to the field $\mathbf{K}(\mathbf{x})$, which happens to coincide with the elastic distortion field in the present circumstances.

We polar decompose $\mathbf{K}$ as

$$
\mathbf{K}=\mathbf{R U},
$$

where $\mathbf{R}$ is a rotation and $\mathbf{U}$ is positive-definite symmetric. To exploit the restriction to small elastic strain we introduce

$$
\boldsymbol{\epsilon}=\mathbf{U}-\mathbf{I}
$$

This yields

$$
\mathbf{E}=\boldsymbol{\epsilon}+\frac{1}{2} \epsilon^{2},
$$

and the restriction is then made explicit by imposing $|\boldsymbol{\epsilon}| \ll 1$, yielding $\mathbf{E}=\boldsymbol{\epsilon}$ to leading order.

Grounds for restricting attention to small elastic strains have already been given. These, however, do not yield any restriction on the rotation field, which may therefore be finite.

Henceforth we use the symbol $\simeq$ to identify expressions that are valid to linear order in the small elastic strain. Thus, for example,

$$
J_{\mathbf{K}} \simeq 1+\operatorname{tr} \boldsymbol{\epsilon}, \quad \mathbf{S} \simeq \mathcal{C}[\boldsymbol{\epsilon}], \quad \boldsymbol{\Pi} \simeq \mathbf{R S R}^{\mathrm{T}} .
$$

We note that the physically accessible lattice field is $\mathbf{d}_{i}=\mathbf{r}_{i}$, where

$$
\mathbf{r}_{i}=\mathbf{R}_{i}+\mathrm{O}(|\boldsymbol{\epsilon}|)
$$

$\mathrm{X}$-ray diffraction experiments are conducted on metallic crystals in which the elastic strain is invariably small, yielding data on lattice orientation (see [10]). Such measurements supply $\mathbf{R}$ directly. Further, it follows from (13) and (19) that the elastic strain yields a significant contribution to the dislocation density only in the case of a curl-free rotation field $\mathbf{R}^{\mathrm{T}}$. This follows from the fact that $\boldsymbol{\alpha}$ is otherwise determined to leading order by the field $\mathbf{R}$.

In the residual stress problem $\kappa_{\mathrm{r}}$ is required to be an equilibrium placement under no body force with zero traction assigned on the entire boundary. Thus,

$$
\operatorname{Div} \boldsymbol{\Pi}=\mathbf{0} \quad \text { in } \kappa_{\mathrm{r}}, \quad \boldsymbol{\Pi n}=\mathbf{0} \quad \text { on } \partial \kappa_{\mathrm{r}} .
$$

This represents an under-determined system for the field $\mathbf{K}(\mathbf{x})$ and hence for the latticevector fields $\mathbf{r}_{i}(\mathbf{x})$. Thus, equilibrium considerations alone do not suffice to determine the residual stress. We return to this issue in Sect. 5, in the context of the two-dimensional theory.

\section{Example: Cubic Crystals}

For cubic crystals the strain-energy function $W$ is invariant under the group of rotations that map a cube to itself. To specify $W$, with reference to $(15)_{1}$, we require the invariant functions that are homogeneous of degree two in the elastic strain, these being common to each of the five subclasses of cubic symmetry. They are

$$
(\operatorname{tr} \boldsymbol{\epsilon})^{2}, \quad \epsilon_{11} \epsilon_{22}+\epsilon_{11} \epsilon_{33}+\epsilon_{22} \epsilon_{33}, \quad \epsilon_{12}^{2}+\epsilon_{13}^{2}+\epsilon_{23}^{2},
$$


where $\epsilon_{i j}=\boldsymbol{\epsilon} \cdot \operatorname{Sym}\left(\mathbf{e}_{i} \otimes \mathbf{e}_{j}\right)$ and $\left\{\mathbf{e}_{i}\right\}$ is an orthonormal basis aligned with the cube axes (i.e., with the elements of the set $\left\{\mathbf{I}_{i}\right\}$ of lattice vectors, or their duals). We emphasize the fact that the orthonormal basis is regarded as remaining fixed at the material point in question, and, for materially uniform bodies, carried over without change to all material points.

Some simplification is achieved by using the deviatoric strain $\bar{\epsilon}=\operatorname{dev} \epsilon$. This yields

$$
\epsilon_{11} \epsilon_{22}+\epsilon_{11} \epsilon_{33}+\epsilon_{22} \epsilon_{33}=\frac{1}{3}(\operatorname{tr} \boldsymbol{\epsilon})^{2}-\frac{1}{2}\left(\bar{\epsilon}_{11}^{2}+\bar{\epsilon}_{22}^{2}+\bar{\epsilon}_{33}^{2}\right),
$$

and so the strain-energy function is expressible in the form [11]

$$
\tilde{W}(\boldsymbol{\epsilon})=\frac{1}{2} C_{1}(\operatorname{tr} \boldsymbol{\epsilon})^{2}+\frac{1}{2} C_{2}\left(\bar{\epsilon}_{11}^{2}+\bar{\epsilon}_{22}^{2}+\bar{\epsilon}_{33}^{2}\right)+C_{3}\left(\epsilon_{12}^{2}+\epsilon_{13}^{2}+\epsilon_{23}^{2}\right),
$$

where $C_{i}, i=1,2,3$, are the (constant) moduli, giving the strain energy as a linear combination of three quadratic forms. (We note in passing that Eq. (89) in reference [5] is missing the factor $1 / 2$ in their expression corresponding to (26) above.) Because these forms are independent and each is positive definite, the basic constitutive hypothesis is met if and only if each $C_{i}>0$. The associated stress is given by $\mathbf{S}=\mathcal{C}[\boldsymbol{\epsilon}]$, where

$$
\begin{aligned}
\mathcal{C}[\boldsymbol{\epsilon}]= & C_{1}(\operatorname{tr} \boldsymbol{\epsilon}) \mathbf{I}+C_{2}\left(\bar{\epsilon}_{11} \mathbf{e}_{1} \otimes \mathbf{e}_{1}+\bar{\epsilon}_{22} \mathbf{e}_{2} \otimes \mathbf{e}_{2}+\bar{\epsilon}_{33} \mathbf{e}_{3} \otimes \mathbf{e}_{3}\right) \\
& +C_{3}\left[\epsilon_{12}\left(\mathbf{e}_{1} \otimes \mathbf{e}_{2}+\mathbf{e}_{2} \otimes \mathbf{e}_{1}\right)+\epsilon_{13}\left(\mathbf{e}_{1} \otimes \mathbf{e}_{3}+\mathbf{e}_{3} \otimes \mathbf{e}_{1}\right)+\epsilon_{23}\left(\mathbf{e}_{2} \otimes \mathbf{e}_{3}+\mathbf{e}_{3} \otimes \mathbf{e}_{2}\right)\right] .
\end{aligned}
$$

\section{Plane Strain in Cubic Crystals}

We confine our further attention to the plane-strain theory for purposes of illustration. This is most easily rendered in Cartesian coordinates, using as the basis the fixed orthonormal triad $\left\{\mathbf{e}_{\alpha}, \mathbf{k}\right\}, \alpha=1,2$, aligned with the specified cubic lattice vectors $\left\{\mathbf{I}_{i}\right\}$ (equivalently, $\left.\left\{\mathbf{I}^{i}\right\}\right)$, where $\mathbf{k}\left(=\mathbf{e}_{3}\right)$ is the unit normal to the considered plane. The non-zero elements of $\mathbf{K}$ in this basis are $K_{\alpha \beta}\left(x_{1}, x_{2}\right)$ and $K_{33}=1$, where $x_{\alpha}$ are the in-plane Cartesian coordinates associated with the lattice vectors. All equations of the model are given to linear order in the small elastic strain.

In the general three-dimensional theory we use (14) together with the standard expression $\operatorname{Curl} \mathbf{v}=e_{j k i} v_{i, k} \mathbf{e}_{j}$, in which the comma is used to denote partial differentiation with respect to $x_{i}$ and $e_{i j k}$ is the usual permutation symbol $\left(e_{123}=+1\right)$, to obtain

$$
\operatorname{Curl}\left(\mathbf{K}^{-1}\right)=e_{i k l} K_{j l, k}^{-1} \mathbf{e}_{i} \otimes \mathbf{e}_{j},
$$

where $K_{j l, k}^{-1}=\left(K_{j l}^{-1}\right)_{, k}$ and $K_{j l}^{-1}=\left(\mathbf{K}^{-1}\right)_{j l}$. The specialization to the present situation is

$$
\operatorname{Curl}\left(\mathbf{K}^{-1}\right)=\varepsilon_{\beta \alpha} K_{\mu \alpha, \beta}^{-1} \mathbf{k} \otimes \mathbf{e}_{\mu},
$$

where $\varepsilon_{\alpha \beta}=e_{3 \alpha \beta}$ is the two-dimensional permutation symbol $\left(\varepsilon_{12}=+1\right)$.

Equation (30) combines with (13) and $\mathbf{K}^{-1} \mathbf{k}=\mathbf{k}$ to generate the dislocation density

$$
\alpha=\mathbf{k} \otimes \mathbf{a},
$$

where $\mathbf{a}=a_{\mu} \mathbf{e}_{\mu}$ is the 2-vector defined by

$$
a_{\mu}=J_{\mathbf{K} \varepsilon_{\beta \alpha}} K_{\mu \alpha, \beta}^{-1} .
$$


To make this explicit we use (19), (20) and (22) with $\boldsymbol{\epsilon}=\epsilon_{\alpha \beta} \mathbf{e}_{\alpha} \otimes \mathbf{e}_{\beta}$ and

$$
\mathbf{R}=\cos \varphi\left(\mathbf{e}_{1} \otimes \mathbf{e}_{1}+\mathbf{e}_{2} \otimes \mathbf{e}_{2}\right)+\sin \varphi\left(\mathbf{e}_{2} \otimes \mathbf{e}_{1}-\mathbf{e}_{1} \otimes \mathbf{e}_{2}\right)+\mathbf{k} \otimes \mathbf{k},
$$

where $\varphi\left(x_{1}, x_{2}\right)$ is the in-plane rotation angle, together with the estimate

$$
\mathbf{K}^{-1} \simeq(\mathbf{I}-\boldsymbol{\epsilon}) \mathbf{R}^{\mathrm{T}},
$$

obtaining

$$
a_{\mu} \simeq \varepsilon_{\beta \alpha}\left[\left(1+\epsilon_{\lambda \lambda}\right) R_{\alpha \mu, \beta}-\epsilon_{\mu \lambda} R_{\alpha \lambda, \beta}-\epsilon_{\mu \lambda, \beta} R_{\alpha \lambda}\right] .
$$

The strain may be eliminated as in [1] by use of the Airy stress function $\chi\left(x_{1}, x_{2}\right)$ in (24); thus,

$$
\Pi_{\alpha \beta}=\varepsilon_{\alpha \lambda} \varepsilon_{\beta \mu} \chi, \lambda \mu,
$$

which satisfies (24) identically in the present circumstances as the remaining stress component, $\Pi_{33}$, is dependent only on $x_{\alpha}$. Here we have used the fact, for cubic crystals with axes oriented as indicated, that the non-zero components of $\mathbf{S}$ in the lattice basis are

$$
S_{11}=A \epsilon_{11}+B \epsilon_{22}, \quad S_{22}=B \epsilon_{11}+A \epsilon_{22}, \quad S_{12}=C \epsilon_{12}, \quad S_{33}=B\left(\epsilon_{11}+\epsilon_{22}\right),
$$

where

$$
A=C_{1}+\frac{2}{3} C_{2}, \quad B=C_{1}-\frac{1}{3} C_{2}, \quad C=C_{3} .
$$

Writing $\mathcal{S}$ for the compliance tensor, we then have $\boldsymbol{\epsilon} \simeq \mathcal{S}[\mathbf{S}]$, where $\mathbf{S} \simeq \mathbf{R}^{\mathrm{T}} \boldsymbol{\Pi} \mathbf{R}$. Explicitly,

$$
\epsilon_{11}=a S_{11}+b S_{22}, \quad \epsilon_{22}=b S_{11}+a S_{22}, \quad \epsilon_{12}=c S_{12},
$$

with

$$
S_{\alpha \beta}=R_{\mu \alpha} R_{\lambda \beta} \Pi_{\mu \lambda}
$$

and

$$
a=A / \Delta, \quad b=-B / \Delta, \quad c=1 / C, \quad \text { where } \quad \Delta=A^{2}-B^{2}=\frac{1}{3} C_{2}^{2}+2 C_{1} C_{2} .
$$

Substitution of (36) and (40) into (39) furnishes the elastic —or lattice-strain $\epsilon_{\alpha \beta}$, i.e., the distortion of $\left\{\mathbf{d}_{i}\right\}$ relative to $\left\{\mathbf{I}_{i}\right\}$ in terms of the scalar fields $\varphi$ and $\chi$.

In the geometrically linear theory, for which the lattice distortion $\mathbf{H}$ is close to the identity (and hence so too the plastic distortion $\mathbf{K}$ in the residual stress problem), the dislocation density field is imposed as data and the stress function is obtained by integrating the linear partial differential equations expressing the incompatibility of the lattice strain [1-3]. However, we know of no rationale justifying the a priori restriction to rotations that are close to the identity, and thus regard the geometrically linear theory as being of limited relevance. In contrast, here the specification of dislocation density as data leads via (33) and (35) to a coupled nonlinear system for the stress function and the lattice orientation field.

We recall that in diffraction experiments it is the lattice orientation field, relative to a specified undistorted lattice, that is typically measured. Its specification is equivalent to that of the rotation field $\mathbf{R}$ in the present circumstances. Only recently have techniques been advanced for the empirical determination of the dislocation density [12]; these make use of 
data for the orientation and (small) elastic strain fields, from which the dislocation density is constructed using (19) with (13), or some equivalent expression. Thus the dislocation density is not measured directly but rather inferred from the measured lattice orientation and strain fields. Measurement of the orientation and elastic strain fields together means that the full distortion field $\mathbf{K}(\mathbf{x})$ is available [13], and hence that the residual stress field is determined in principle via constitutive equations such as $(22)_{2,3}$. Thus experiments that yield the dislocation density also yield the residual stress to the same degree, at least in principle, granted the availability of the elastic moduli. As a practical matter, this state of affairs obviates the classical residual stress problem, in which dislocation density is specified as data and the stress is regarded as a field to be derived, since the experimental information needed to specify the data also furnish the solution.

The rotation field appears to be the variable that is most reliably known from the experimental point of view (see [12], p. 1345). Presumably this is due to the fact that the elastic strain is nearly negligible in practice and thus more sensitive to errors in measurement. For this reason we regard the rotation field, rather than the dislocation density, as assigned data. If the dislocation density were also specified then (35) would furnish an overdetermined system for the Airy stress function. The integrability condition for this system amounts to an $a$ priori relationship involving the components $a_{\mu}$, implying that the latter may not be specified independently. Alternatively, the field $\chi$ may be specified, subject to an appropriate boundary condition, and the dislocation density then evaluated using (35).

The traction data $(24)_{2}$ impose restrictions on the derivatives of $\chi$ on the boundary curve $\partial \kappa$ of the (two-dimensional) domain $\kappa$, where $\kappa_{\mathrm{r}}=\kappa \times[0,1]$ and $\partial \kappa_{\mathrm{r}}=\partial \kappa \times[0,1]$ for a slab of unit depth, say. Using (36) with $n_{\beta}=\varepsilon_{\beta \lambda} x_{\lambda}^{\prime}$, where $x_{\alpha}(s)$ is the arclength parametrization of $\partial \kappa$ and $(\cdot)^{\prime}=\mathrm{d}(\cdot) / \mathrm{d} s$, we obtain

$$
0=\Pi_{\alpha \beta} n_{\beta}=\varepsilon_{\alpha \varphi} \chi, \varphi \lambda x_{\lambda}^{\prime}=\varepsilon_{\alpha \varphi}(\chi, \varphi)^{\prime} \quad \text { on } \partial \kappa,
$$

in which use has been made of $\varepsilon_{\beta \mu} \varepsilon_{\beta \lambda}=\delta_{\mu \lambda}$ (the Kronecker delta). This is equivalent to

$$
\nabla \chi=\mathbf{c} \quad \text { on } \partial \kappa,
$$

in which $\nabla$ is the (two-dimensional) gradient and $\mathbf{c}$ is a constant vector. If $\partial \kappa$ consists of disjoint curves, then the constant vector may vary from one curve to another. In the present interpretation in which the rotation field is assigned, an Airy stress function satisfying (43) is selected and the associated dislocation density is obtained directly from (35). Thus the latter is regarded as a derived quantity generated by the stress (or lattice strain), granted the availability of the orientation field. This is in keeping with the protocol of contemporary experiments (see, for example, [12]).

As previously mentioned, lattice strain makes a non-negligible contribution to the dislocation density only if the rotation field satisfies a zero-curl condition. In the present setting this condition is $\varepsilon_{\beta \alpha} R_{\alpha \mu, \beta}=0$ (cf. (35)). Using (33) we reduce this to $\nabla \varphi=\mathbf{0}$, in which case (35) furnishes the connection

$$
a_{\mu} \simeq-\varepsilon_{\beta \alpha} \epsilon_{\mu \lambda, \beta} R_{\alpha \lambda}
$$

yielding the dislocation distribution associated with a particular Airy stress function. Alternatively, lattice strain may be neglected in the evaluation of dislocation density whenever the rotation field is non-uniform. 


\section{References}

1. Kröner, E.: Continuum theory of defects. In: Balian, R., et al. (eds.) Les Houches, Session XXXV, 1980-Physique des défauts, pp. 215-315. North-Holland, Amsterdam (1981)

2. Teodosiu, C.: Elastic Models of Crystal Defects. Springer, Berlin (1982)

3. Lardner, R.W.: Mathematical Theory of Dislocations and Fracture. University of Toronto Press, Toronto (1974)

4. Gupta, A., Steigmann, D.J., Stölken, J.S.: On the evolution of plasticity and incompatibility. Math. Mech. Solids 12, 583-610 (2007)

5. Gupta, A., Steigmann, D.J., Stölken, J.S.: Aspects of the phenomenological theory of elastic-plastic deformation. J. Elast. 104, 249-266 (2011)

6. Ogden, R.W.: Non-linear Elastic Deformations. Dover, New York (1997)

7. Cermelli, P., Gurtin, M.E.: On the characterization of geometrically necessary dislocations in finite plasticity. J. Mech. Phys. Solids 49, 1539-1568 (2001)

8. Cullity, B.: Elements of X-Ray Diffraction. Addison-Wesley, Reading (1978)

9. Epstein, M., Elzanowski, M.: Material Inhomogeneities and Their Evolution. Springer, Berlin (2007)

10. Gurtin, M.E., Fried, E., Anand, L.: Mechanics and Thermodynamics of Continua. Cambridge University Press, Cambridge (2010)

11. Ericksen, J.L.: Constitutive theory for some constrained elastic crystals. Int. J. Solids Struct. 22, 951-964 (1986)

12. Larson, B.C., El-Azab, A., Yang, W., Tischler, J.Z., Liu, W., Ice, G.E.: Experimental characterization of the mesoscale dislocation density tensor. Philos. Mag. 87, 1327-1347 (2007)

13. Edmiston, J., Barton, N., Bernier, J., Johnson, G., Steigmann, D.J.: Precision of lattice strain and orientation measurements using high energy monochromatic X-ray diffraction. J. Appl. Crystallogr. 44, 299-312 (2011) 